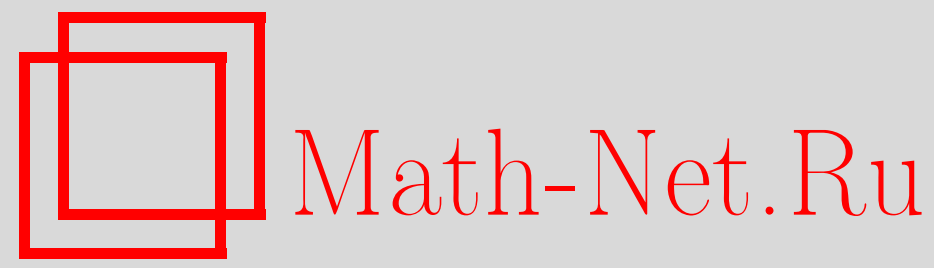

М. Ф. Кулагина, В. И. Иванова, Первая основная задача теории упругости для области, состоящей из полосы и полуплоскости, Вестн. Сам. гос. техн. ун-та. Сер. Физ.-мат. науки, 2003, выпуск 19, 89-96

DOI: https://doi.org/10.14498/vsgtu146

Использование Общероссийского математического портала Math-Net.Ru подразумевает, что вы прочитали и согласны с пользовательским соглашением

http: //www.mathnet.ru/rus/agreement

Параметры загрузки:

IP: 54.210 .77 .194

26 апреля 2023 г., 15:43:03 


\section{ПЕРВАЯ ОСНОВНАЯ ЗАДАЧА ТЕОРИИ УПРУГОСТИ ДЛЯ ОБЛАСТИ, СОСТОЯЩЕЙ ИЗ ПОЛОСЫ И ПОЛУПЛОСКОСТИ}

Приведено решение первой основной задачи теории упругости для области, состоящей из однородно-изотропных полосы и полуплоскости, на общей границе которых заданы условия контакта с трением. Решение построено в пространстве почтипериодических в смысле Бора напряжений и перемещений с помощью обобщенного дискретного преобразования Фурье. Искомые функции представлены в виде рядов Фурье, сходящихся абсолютно и равномерно.

Почтипериодическим (п.п.) полиномом называется функция $p(t),-\infty<t<\infty$, являющаяся линейной комбинацией функций вида $e^{i \lambda t}$, где $\lambda \in R$. Обозначим через $\Pi_{C}$ замыкание по норме $L_{\infty}(-\infty, \infty)$ множества всех п. п. полиномов. Множество $\Pi_{C}$ является подалгеброй $L_{\infty}(-\infty, \infty)$, состоящей из всех п.П. функций по Бору [1,2]. Через $\Pi_{W}$ обозначим множество $\Pi_{C}$, состоящее из функций $A(t)$ вида

$$
A(t)=\sum_{n=1}^{\infty} a_{n} e^{i \lambda_{n} t}
$$

удовлетворяющих условию $\sum_{n=1}^{\infty}\left|a_{n}\right|<\infty$. Множество $\Pi_{W}$ является банаховой алгеброй [3].

Каждой функции $A(t)$ из $\Pi_{W}$ поставим в соответствие функцию

$$
a(\lambda)=M\left\{A(t) e^{-i \lambda t}\right\}=\lim _{T \rightarrow \infty} \frac{1}{2 T} \int_{-T}^{T} A(t) e^{-i \lambda t} d t .
$$

Такая функция существует и может быть отличной от нуля не более чем для счетного множества значений $\lambda: \lambda_{1}, \lambda_{2}, \ldots: a\left(\lambda_{n}\right)=a_{n} \neq 0$ [4]. Таким образом, каждой функции из $\Pi_{W}$ поставлена в соответствие функция $a(\lambda)$ или последовательность пар $a(\lambda)=\left\{\left(a_{1}, \lambda_{1}\right),\left(a_{2}, \lambda_{2}\right), \ldots\right\}$, где $a_{n} \in C, \lambda_{n} \in R$.

Если $A(t) \in \Pi_{W}$, то соответствующая этой функции последовательность $\left\{a_{n}\right\} \in l_{1}$ (будем говорить, что $\left.a(\lambda) \in l_{1}\right)$. И наоборот, для каждой функции $a(\lambda) \in l_{1}$ существует функция $A(t)$, для которой выполнено (2), и $A(t)$ имеет вид (1). Ряд (1) сходится абсолютно и равномерно при $-\infty<t<\infty$. Следовательно, установлено взаимнооднозначное соответствие между функциями из $\Pi_{W}$ и двумерными последовательностями $a(\lambda) \in l_{1}$. При этом мы считаем, что две последовательности $a(\lambda), b(\lambda) \in l_{1}$ совпадают, если им соответствуют одни и те же функции, т.е. последовательность $a(\lambda)$ не изменится, если к ней добавить счетное множество пар вида $(0, \lambda)$.

Равенство (1), которое последовательности $a(\lambda) \in l_{1}$ ставит в соответствие функцию $A(t) \in \Pi_{W}$, назовем обобщенным дискретным преобразованием Фурье (ОДФ). Равенство (2) производит обратное преобразование. Последовательность $a(\lambda)$ - оригинал, функция $A(t)-$ изображение. ОДФ будем изображать символами $A(t)=W_{0} a(\lambda), a(\lambda)=W_{0}^{-1} A(t)$.

Коэффициенты последовательности $a(\lambda)$ могут зависеть от параметра $y: a(\lambda, y)=$ $\left\{\left(a_{1}(y), \lambda_{1}\right),\left(a_{2}(y), \lambda_{2}\right), \ldots\right\} \quad y \in[a, b]$. Если существует последовательность положительных чисел $\left\{a_{n}\right\} \in l_{1}$, такая, что $\left|a_{n}(y)\right| \leq a_{n}$, то функции $A(t, y)=W_{0} a(\lambda, y) \in \Pi_{W}$ на каждой горизонтальной прямой полосе $a \leq \operatorname{Im} z \leq b \quad(z=t+i y)$. Будем считать, что функция $A(t, y) \in \Pi_{W}^{y}$ $(y \in[a, b])$.

Постановка задачи. Область, состоящая из однородно-изотропной упругой полосы $(y \in[0 ; 1],-\infty<x<\infty)$ и такой же полуплоскости $(y \in(-\infty ; 0],-\infty<x<\infty)$, находится в равновесии. На прямой соединения $y=0$ заданы условия контакта с трением:

$$
v^{(1)}(x, 0)=v^{(2)}(x, 0), \sigma_{y}^{(1)}(x, 0)=\sigma_{y}^{(2)}(x, 0), \tau_{x y}^{(1)}(x, 0)=\tau_{x y}^{(2)}(x, 0), \tau_{x y}^{(1)}(x, 0)=K \sigma_{y}^{(1)}(x, 0),
$$


где $K=$ const - коэффициент трения.

На прямой $y=1$ заданы граничные условия первого рода:

$$
\sigma_{y}^{(1)}(x, 1)=f(x), \tau_{x y}^{(1)}(x, 1)=g(x) .
$$

Требуется найти напряжения $\sigma_{x}^{(j)}(x, y), \sigma_{y}^{(j)}(x, y), \tau_{x y}^{(j)}(x, y)$ и перемещения $u^{(j)}(x, y)$, $v^{(j)}(x, y)(j=1,2)$ в заданной области (здесь и далее верхний индекс 1 обозначает функции в полосе, индекс 2 - функции в полуплоскости).

Будем считать, что заданные функции $f(x)$ и $g(x)$ принадлежат $\Pi_{W}$ и представимы в виде рядов

$$
f(x)=f_{0}+\sum_{\lambda \neq 0} f(\lambda) e^{i \lambda x} ; g(x)=g_{0}+\sum_{\lambda \neq 0} g(\lambda) e^{i \lambda x},
$$

причем последовательности $f(\lambda)$ и $g(\lambda)$ не сгущаются к нулю.

Решение задачи будем искать в таком классе, чтобы искомые механические параметры (напряжения и перемещения) принадлежали классу $\Pi_{W}^{y}(y \in[0 ; 1])$ в полосе и классу $\Pi_{W}^{y}(y \in(-\infty ; 0])$ в полуплоскости, причем все искомые напряжения и перемещения в полуплоскости при $y \rightarrow-\infty$ равномерно стремятся к нулю.

P e шI е н и е. Воспользуемся методом, основанным на применении функций ПапковичаНейбера [5]. Искомые механические параметры $\sigma_{x}^{(j)}(x, y), \sigma_{y}^{(j)}(x, y), \tau_{x y}^{(j)}(x, y), u^{(j)}(x, y)$, $v^{(j)}(x, y) \quad(j=1,2)$ выражаются через пары гармонических функций $\Phi_{0}^{(j)}(x, y)$ и $\Phi_{2}^{(j)}(x, y)$ по формулам

$$
\begin{gathered}
2 G^{(j)} u^{(j)}(x, y)=-\frac{\partial F^{(j)}(x, y)}{\partial x} ; \\
2 G^{(j)} v^{(j)}(x, y)=-\frac{\partial F^{(j)}(x, y)}{\partial y}+4\left(1-v^{(j)}\right) \Phi_{2}^{(j)}(x, y) \\
\sigma_{x}^{(j)}(x, y)=-\frac{\partial^{2} \Phi_{0}^{(j)}(x, y)}{\partial x^{2}}+2 v^{(j)} \frac{\partial \Phi_{2}^{(j)}(x, y)}{\partial y}-y \frac{\partial^{2} \Phi_{2}^{(j)}(x, y)}{\partial x^{2}} ; \\
\tau_{x y}^{(j)}(x, y)=-\frac{\partial}{\partial x}\left[\frac{\partial \Phi_{0}^{(j)}(x, y)}{\partial y}+y \frac{\partial \Phi_{2}^{(j)}(x, y)}{\partial y}\right]+\left(1-2 v^{(j)}\right) \frac{\partial \Phi_{2}^{(j)}(x, y)}{\partial x} ; \\
\sigma_{y}^{(j)}(x, y)=\frac{\partial}{\partial y}\left[2\left(1-v^{(j)}\right) \Phi_{2}^{(j)}(x, y)-\frac{\partial \Phi_{0}^{(j)}(x, y)}{\partial y}\right]-y \frac{\partial^{2} \Phi_{2}^{(j)}(x, y)}{\partial y^{2}},
\end{gathered}
$$

где $F^{(j)}(x, y)=\Phi_{0}^{(j)}(x, y)+y \Phi_{2}^{(j)}(x, y) ; \boldsymbol{v}^{(j)}-$ коэффициент Пуассона; $G^{(j)}$ - модуль сдвига, $j=1,2$, их мы будем считать заданными постоянными величинами.

Чтобы искомые напряжения и перемещения принадлежали нужным классам, гармонические функции $\Phi_{0}^{(j)}(x, y)$ и $\Phi_{2}^{(j)}(x, y) \quad(j=1,2)$ и их частные производные до второго порядка включительно должны принадлежать классу $\Pi_{W}^{y}(y \in[0 ; 1])$ в полосе и классу $\Pi_{W}^{y}(y \in(-\infty ; 0])$ в полуплоскости.

Применяя к уравнению Лапласа преобразование $W_{0}^{-1}$ и учитывая его свойства, нетрудно показать, что эти функции имеют следующую структуру: в полосе

$$
\begin{aligned}
& \Phi_{0}^{(1)}(x, y)=a_{0}^{(1)}+a_{1}^{(1)} y+\sum_{\lambda \neq 0}\left(a^{(1)}(\lambda) e^{\lambda y}+b^{(1)}(\lambda) e^{-\lambda y}\right) e^{i \lambda x} \\
& \Phi_{2}^{(1)}(x, y)=c_{0}^{(1)}+c_{1}^{(1)} y+\sum_{\lambda \neq 0}\left(c^{(1)}(\lambda) e^{\lambda y}+d^{(1)}(\lambda) e^{-\lambda y}\right) e^{i \lambda x},
\end{aligned}
$$

в полуплоскости

$$
\begin{aligned}
& \Phi_{0}^{(2)}(x, y)=a_{0}^{(2)}+\sum_{\lambda \neq 0} a^{(2)}(\lambda) e^{|\lambda| y} e^{i \lambda x} ; \\
& \Phi_{2}^{(2)}(x, y)=c_{0}^{(2)}+\sum_{\lambda \neq 0} c^{(2)}(\lambda) e^{|\lambda| y} e^{i \lambda x} .
\end{aligned}
$$


Подставляя (7) и (8) в формулы (6), получим выражение искомых функций в виде рядов Фурье с неизвестными коэффициентами $a^{(1)}(\lambda), b^{(1)}(\lambda), c^{(1)}(\lambda), d^{(1)}(\lambda), a^{(2)}(\lambda), c^{(2)}(\lambda)$. В полосе

$$
\begin{aligned}
2 G^{(1)} u^{(1)}(x, y)= & -\sum_{\lambda \neq 0} i \lambda\left(a^{(1)}(\lambda) e^{\lambda y}+b^{(1)}(\lambda) e^{-\lambda y}\right) e^{i \lambda x}-y \sum_{\lambda \neq 0} i \lambda\left(c^{(1)}(\lambda) e^{\lambda y}+d^{(1)}(\lambda) e^{-\lambda y}\right) e^{i \lambda x} \\
2 G^{(1)} v^{(1)}(x, y)= & \kappa^{(1)} c_{0}^{(1)}-a_{1}^{(1)}+\kappa^{(1)} c_{1}^{(1)} y-c_{1}^{(1)} y-\sum_{\lambda \neq 0} \lambda\left(a^{(1)}(\lambda) e^{\lambda y}-b^{(1)}(\lambda) e^{-\lambda y}\right) e^{i \lambda x}+ \\
& +\sum_{\lambda \neq 0}\left(c^{(1)}(\lambda)\left[\kappa^{(1)}-\lambda y\right] e^{\lambda y}+d^{(1)}(\lambda)\left[\kappa^{(1)}+\lambda y\right] e^{-\lambda y}\right) e^{i \lambda x} \\
\sigma_{x}^{(1)}(x, y)= & 2 v^{(1)} c_{1}^{(1)}+\sum_{\lambda \neq 0} \lambda^{2}\left(a^{(1)}(\lambda) e^{\lambda y}+b^{(1)}(\lambda) e^{-\lambda y}\right) e^{i \lambda x}+ \\
& +\sum_{\lambda \neq 0} \lambda\left(c^{(1)}(\lambda)\left[2 v^{(1)}+\lambda y\right] e^{\lambda y}+d^{(1)}(\lambda)\left[-2 v^{(1)}+\lambda y\right] e^{-\lambda y}\right) e^{i \lambda x} \\
\sigma_{y}^{(1)}(x, y)= & 2\left(1-v^{(1)}\right) c_{1}^{(1)}-\sum_{\lambda \neq 0} \lambda^{2}\left(a^{(1)}(\lambda) e^{\lambda y}+b^{(1)}(\lambda) e^{-\lambda y}\right) e^{i \lambda x}+ \\
& +\sum_{\lambda \neq 0} \lambda\left(c^{(1)}(\lambda)\left[2-2 v^{(1)}-\lambda y\right] e^{\lambda y}-d^{(1)}(\lambda)\left[2-2 v^{(1)}+\lambda y\right] e^{-\lambda y}\right) e^{i \lambda x} \\
\tau_{x y}^{(1)}(x, y)= & -\sum_{\lambda \neq 0} i \lambda^{2}\left(a^{(1)}(\lambda) e^{\lambda y}-b^{(1)}(\lambda) e^{-\lambda y}\right) e^{i \lambda x}+ \\
& +\sum_{\lambda \neq 0} i \lambda\left(c^{(1)}(\lambda)\left[1-2 v^{(1)}-\lambda y\right] e^{\lambda y}+d^{(1)}(\lambda)\left[1-2 v^{(1)}+\lambda y\right] e^{-\lambda y}\right) e^{i \lambda x}
\end{aligned}
$$

в полуплоскости

$$
\begin{aligned}
& 2 G^{(2)} u^{(2)}(x, y)=-\sum_{\lambda \neq 0} i \lambda a^{(2)}(\lambda) e^{|\lambda| y} e^{i \lambda x}-y \sum_{\lambda \neq 0} i \lambda c^{(2)}(\lambda) e^{|\lambda| y} e^{i \lambda x} ; \\
& 2 G^{(2)} v^{(2)}(x, y)=c_{0}^{(2)} \kappa^{(2)}-\sum_{\lambda \neq 0}|\lambda| a^{(2)}(\lambda) e^{|\lambda| y} e^{i \lambda x}+\sum_{\lambda \neq 0}\left(\kappa^{(2)}-|\lambda| y\right) c^{(2)}(\lambda) e^{|\lambda| y} e^{i \lambda x} \\
& \sigma_{x}^{(2)}(x, y)=\sum_{\lambda \neq 0}\left(\lambda^{2}+2 v^{(2)}|\lambda|\right) a^{(2)}(\lambda) e^{|\lambda| y} e^{i \lambda x}+y \sum_{\lambda \neq 0} \lambda^{2} c^{(2)}(\lambda) e^{|\lambda| y} e^{i \lambda x} ; \\
& \tau_{x y}^{(2)}(x, y)=-\sum_{\lambda \neq 0} i \lambda|\lambda| a^{(2)}(\lambda) e^{|\lambda| y} e^{i \lambda x}+\sum_{\lambda \neq 0} i \lambda\left(-y|\lambda|+1-2 v^{(2)}\right) c^{(2)}(\lambda) e^{|\lambda| y} e^{i \lambda x} \\
& \sigma_{y}^{(2)}(x, y)=-\sum_{\lambda \neq 0} \lambda^{2} a^{(2)}(\lambda) e^{|\lambda| y} e^{i \lambda x}-\sum_{\lambda \neq 0}\left(y \lambda^{2}-2\left(1-v^{(2)}\right)|\lambda|\right) c^{(2)}(\lambda) e^{|\lambda| y} e^{i \lambda x}
\end{aligned}
$$

где $\kappa^{(j)}=3-4 v^{(j)}, j=1,2$.

Используя условия контакта с трением (3) и граничные условия (4), получим уравнения для нахождения неизвестных коэффициентов $a^{(1)}(\lambda), b^{(1)}(\lambda), c^{(1)}(\lambda), d^{(1)}(\lambda), a^{(2)}(\lambda)$, $c^{(2)}(\lambda)$. При $\lambda=0$ имеем

$$
\frac{\kappa^{(1)} c_{0}^{(1)}-a_{1}^{(1)}}{2 G^{(1)}}=\frac{\kappa^{(2)} c_{0}^{(2)}}{2 G^{(2)}} ; 2\left(1-v^{(1)}\right) c_{1}^{(1)}=f_{0}, 2\left(1-v^{(1)}\right) c_{1}^{(1)}=0, g_{0}=0 .
$$

Из этого представления видно, что для разрешимости задачи необходимо (а далее будет показано, что и достаточно), чтобы $f_{0}=g_{0}=0$, т.е.

$$
\lim _{T \rightarrow \infty} \frac{1}{2 T} \int_{-T}^{T} f(x) d x=\lim _{T \rightarrow \infty} \frac{1}{2 T} \int_{-T}^{T} g(x) d x=0,
$$

тогда

$$
c_{1}^{(1)}=0, c_{0}^{(2)}=\frac{G^{(2)}\left(\kappa^{(1)} c_{0}^{(1)}-a_{1}^{(1)}\right)}{G^{(1)} \kappa^{(2)}} .
$$

Если при постановке задачи считать заданным

$$
\kappa^{(1)} c_{0}^{(1)}-a_{1}^{(1)}=2 G^{(1)} \cdot \lim _{T \rightarrow \infty} \frac{1}{2 T} \int_{-T}^{T} v^{(1)}(x, 0) d x,
$$

то коэффициенты при $\lambda=0$ определяются однозначно.

При $\lambda \neq 0$ получим систему из 6 линейных уравнений с шестью неизвестными, матричная запись которой имеет вид 


$$
M(\lambda) \cdot s(\lambda)=m(\lambda)
$$

где

$$
M(\lambda)=\left[\begin{array}{cccccc}
-\lambda e^{\lambda} & -\lambda e^{-\lambda} & \left(2-2 v^{(1)}-\lambda\right) e^{\lambda} & -\left(2-2 v^{(1)}+\lambda\right) e^{-\lambda} & 0 & 0 \\
-\lambda e^{\lambda} & \lambda e^{-\lambda} & \left(1-2 v^{(1)}-\lambda\right) e^{\lambda} & \left(1-2 v^{(1)}+\lambda\right) e^{-\lambda} & 0 & 0 \\
-\frac{\lambda}{G^{(1)}} & \frac{\lambda}{G^{(1)}} & \frac{\kappa^{(1)}}{G^{(1)}} & \frac{\kappa^{(1)}}{G^{(1)}} & \frac{|\lambda|}{G^{(2)}} & -\frac{\kappa^{(2)}}{G^{(2)}} \\
-\lambda^{2} & -\lambda^{2} & 2 \lambda\left(1-v^{(1)}\right) & -2 \lambda\left(1-v^{(1)}\right) & \lambda^{2} & -2|\lambda|\left(1-v^{(2)}\right) \\
-\lambda & \lambda & 1-2 v^{(1)} & 1-2 v^{(1)} & |\lambda| & -1+2 v^{(2)} \\
(K-i) \lambda & (K+i) \lambda & i\left(1-2 v^{(1)}\right)-K\left(2-2 v^{(1)}\right) & i\left(1-2 v^{(1)}\right)+K\left(2-2 v^{(1)}\right) & 0 & 0
\end{array}\right]
$$

$s(\lambda)=\operatorname{col}\left(a^{(1)}(\lambda), b^{(1)}(\lambda), c^{(1)}(\lambda), d^{(1)}(\lambda), a^{(2)}(\lambda), c^{(2)}(\lambda)\right), m(\lambda)=\operatorname{col}\left(\frac{f(\lambda)}{\lambda}, \frac{g(\lambda)}{i \lambda}, 0,0,0,0\right)$.

Система (15) имеет единственное решение $s(\lambda)$, если ее определитель $\Delta(\lambda)=\operatorname{det}(M(\lambda))$ отличен от нуля при $0<|\lambda|<\infty$. Учитывая линейную независимость функций $1, \lambda, \lambda^{2}, e^{\lambda}, e^{-\lambda}$, $\lambda e^{\lambda}, \lambda e^{-\lambda}$, легко показать, что строки определителя $\Delta(\lambda)$ линейно независимы. Следовательно, $\Delta(\lambda)$ не равен нулю при $0<|\lambda|<\infty$, поэтому система (15) имеет единственное решение, которое можно найти, например, по формулам Крамера.

Из структуры коэффициентов $a^{(1)}(\lambda), b^{(1)}(\lambda), c^{(1)}(\lambda), d^{(1)}(\lambda), a^{(2)}(\lambda), c^{(2)}(\lambda)$ следует, что если заданные функции $f(x)$ и $g(x)$ принадлежат $\Pi_{W}$, то ряды в формулах (9) и (10) сходятся абсолютно и равномерно.

Итак, справедливо утверждение.

Т е о р е м а. Пусть функции $f(x)$ и $g(x)$ принадлежат $\Pi_{W}$ и представимы в виде (5), причем последовательности $f(\lambda)$ и $g(\lambda)$ не сгущаются к нулю, и пусть выполнено условие (12) и задано (14). Тогда первая основная задача теории упругости (4) для горизонтальной однородно-изотропной упругой полосы $y \in[0 ; 1],-\infty<x<\infty$ и такой же полуплоскости $y \in(-\infty ; 0],-\infty<x<\infty$, разделенных прямой $y=0$, на которой заданы условия контакта с трением (3), имеет единственное решение, где коэффициенты, входящие в формулы (9) и (10), находятся из (13) и (15).

Данная задача относилась к случаю плоской деформации. Для плоского напряженного состояния во всех формулах следует заменить $\boldsymbol{v}^{(j)}$ на $\frac{\boldsymbol{v}^{(j)}}{1+\boldsymbol{v}^{(j)}}, j=1,2$.

П $\boldsymbol{p} \boldsymbol{u} \boldsymbol{м} \boldsymbol{e} \boldsymbol{p}$. Рассмотрим плоское деформационное состояние однородноизотропной упругой полосы $\left(y \in[0 ; 1], \quad-\infty<x<\infty, v^{(1)}=0,3, \quad G^{(1)}=7 \cdot 10^{10}\right)$ и такой же полуплоскости $\left(y \in(-\infty ; 0],-\infty<x<\infty, v^{(1)}=0,4, G^{(1)}=9 \cdot 10^{10}\right)$, на общей границе которых заданы условия контакта с трением (3), где $K=1,5$. Граничные функции в (4) заданы в следующем виде:

$$
\begin{gathered}
f(x)=\cos x-2 \sin \sqrt{2} x+\cos \pi x=\frac{e^{i x}+e^{-i x}}{2}-\frac{e^{i \sqrt{2} x}-e^{-i \sqrt{2} x}}{i}+\frac{e^{i \pi x}+e^{-i \pi x}}{2} \\
g(x)=-3 \sin x+2 \cos \sqrt{2} x+2 \sin \pi x=-3 \cdot \frac{e^{i x}-e^{-i x}}{2 i}+e^{i \sqrt{2} x}+e^{-i \sqrt{2} x}+\frac{e^{i \pi x}-e^{-i \pi x}}{i} .
\end{gathered}
$$

Для однозначности решения зададим в (14) $\lim _{T \rightarrow \infty} \frac{1}{2 T} \int_{-T}^{T} v^{(1)}(x, 0) d x=0$.

Решив систему (15) при $\lambda= \pm 1, \pm \sqrt{2}, \pm \pi$, найдем соответствующие им коэффициенты $a^{(1)}(\lambda), b^{(1)}(\lambda), c^{(1)}(\lambda), d^{(1)}(\lambda), a^{(2)}(\lambda), c^{(2)}(\lambda)$ и, подставив их в формулы (9) и (10), получим искомые функции в полосе и полуплоскости:

$$
\begin{aligned}
u^{(1)}(x, y) & =-0,510 \cdot 10^{-11} e^{y} \sin x+0,165 \cdot 10^{-12} e^{y} \cos x-0,625 \cdot 10^{-11} e^{-y} \sin x+ \\
& +0,332 \cdot 10^{-11} e^{-y} \cos x+0,101 \cdot 10^{-11} e^{\sqrt{2} y} \sin \sqrt{2} x-0,213 \cdot 10^{-11} e^{\sqrt{2} y} \cos \sqrt{2} x+ \\
& +0,205 \cdot 10^{-10} e^{-\sqrt{2} y} \sin \sqrt{2} x+0,73 \cdot 10^{-11} e^{-\sqrt{2} y} \cos \sqrt{2} x-0,614 \cdot 10^{-12} e^{\pi y} \sin \pi x-
\end{aligned}
$$


$-0,360 \cdot 10^{-13} e^{\pi y} \cos \pi x-0,421 \cdot 10^{-12} e^{-\pi y} \sin \pi x-0,249 \cdot 10^{-11} e^{-\pi y} \cos \pi x-$ $-0,460 \cdot 10^{-11} y e^{y} \sin x-0,203 \cdot 10^{-12} y e^{y} \cos x+0,457 \cdot 10^{-11} y e^{-y} \sin x-$ $-0,214 \cdot 10^{-11} y e^{-y} \cos x-0,169 \cdot 10^{-11} y e^{\sqrt{2} y} \sin \sqrt{2} x+0,618 \cdot 10^{-11} y e^{\sqrt{2} y} \cos \sqrt{2} x-$ $-0,187 \cdot 10^{-10} y e^{-\sqrt{2} y} \sin \sqrt{2} x-0,570 \cdot 10^{-11} y e^{-\sqrt{2} y} \cos \sqrt{2} x+0,930 \cdot 10^{-12} y e^{\pi y} \sin \pi x+$ $+0,483 \cdot 10^{-13} y e^{\pi y} \cos \pi x+0,573 \cdot 10^{-12} y e^{-\pi y} \sin \pi x+0,513 \cdot 10^{-11} y e^{-\pi y} \cos \pi x$;

$v^{(1)}(x, y)=-0,925 \cdot 10^{-13} e^{-\pi y} \cos \pi x-0,637 \cdot 10^{-13} e^{\pi y} \sin \pi x+0,460 \cdot 10^{-11} y e^{y} \cos x-$

$-0,203 \cdot 10^{-12} y e^{y} \sin x+0,214 \cdot 10^{-12} y e^{-y} \sin x+0,169 \cdot 10^{-11} y e^{\sqrt{2} y} \cos \sqrt{2} x-$ $-0,187 \cdot 10^{-10} y e^{-\sqrt{2} y} \cos \sqrt{2} x+0,570 \cdot 10^{-11} y e^{-\sqrt{2} y} \sin \sqrt{2} x-0,930 \cdot 10^{-12} y e^{\pi y} \cos \pi x+$ $+0,483 \cdot 10^{-13} y e^{\pi y} \sin \pi x+0,618 \cdot 10^{-11} y e^{\sqrt{2} y} \sin \sqrt{2} x+0,457 \cdot 10^{-11} y e^{-y} \cos x+$ $+0,573 \cdot 10^{-12} y e^{-\pi y} \cos \pi-0,513 \cdot 10^{-11} y e^{-\pi y} \sin \pi x-0,332 \cdot 10^{-11} e^{-\sqrt{2} y} \cos \sqrt{2} x+$ $+0,253 \cdot 10^{-11} e^{-\sqrt{2} y} \sin \sqrt{2} x+0,115 \cdot 10^{-11} e^{\pi y} \cos \pi x-0,455 \cdot 10^{-12} e^{-\pi y} \sin \pi x+$ $+0,534 \cdot 10^{-12} e^{-y} \sin x-0,317 \cdot 10^{-11} e^{y} \cos x+0,530 \cdot 10^{-12} e^{y} \sin x+$ $+0,197 \cdot 10^{-11} e^{-y} \cos x-0,316 \cdot 10^{-11} e^{\sqrt{2} y} \cos \sqrt{2} x-0,999 \cdot 10^{-11} e^{\sqrt{2} y} \sin \sqrt{2} x ;$

$\sigma_{x}^{(1)}(x, y)=-0,233 e^{-\pi y} \cos \pi x+0,012 e^{\pi y} \sin \pi x-0,644 y e^{y} \cos x+0,028 y e^{y} \sin x+$ $+0,639 y e^{-y} \cos x+0,300 y e^{-y} \sin x-0,335 y e^{\sqrt{2} y} \cos \sqrt{2} x-1,22 y e^{\sqrt{2} y} \sin \sqrt{2} x-$ $-3,71 y e^{-\sqrt{2} y} \cos \sqrt{2} x+1,13 y e^{-\sqrt{2} y} \sin \sqrt{2} x+0,409 y e^{\pi y} \cos \pi x-0,021 y e^{\pi y} \sin \pi x+$ $+0,252 y e^{-\pi y} \cos \pi x-2,26 y e^{-\pi y} \sin \pi x+5,64 e^{-\sqrt{2} y} \cos \sqrt{2} x-1,42 e^{-\sqrt{2} y} \sin \sqrt{2} x-$ $-0,192 e^{\pi y} \cos \pi x+1,53 e^{-\pi y} \sin \pi x-0,645 e^{-y} \sin x-1,10 e^{y} \cos x-0,006 e^{y} \sin x-$ $-1,26 e^{-y} \cos x+0,057 e^{\sqrt{2} y} \cos \sqrt{2} x-0,098 e^{\sqrt{2} y} \sin \sqrt{2} x$;

$\tau_{x y}^{(1)}(x, y)=0,806 e^{-\pi y} \cos \pi x-0,322 e^{\pi y} \sin \pi x-0,644 y e^{y} \sin x-0,028 y e^{y} \cos x-$

$-0,639 y e^{-y} \sin x+0,300 y e^{-y} \cos x-0,335 y e^{\sqrt{2} y} \sin \sqrt{2} x+1,22 y e^{\sqrt{2} y} \cos \sqrt{2} x+$ $+3,71 y e^{-\sqrt{2} y} \sin \sqrt{2} x++1,13 y e^{-\sqrt{2} y} \cos \sqrt{2} x+0,409 y e^{\pi y} \sin \pi x+0,021 y e^{\pi y} \cos \pi x-$ $-0,252 y e^{-\pi y} \sin \pi x-2,26 y e^{-\pi y} \cos \pi x-0,617 e^{-\sqrt{2} y} \cos \sqrt{2} x-3,01 e^{-\sqrt{2} y} \sin \sqrt{2} x-$ $-0,019 e^{\pi y} \cos \pi x+0,153 e^{-\pi y} \sin \pi x+0,619 e^{-y} \sin x+0,035 e^{y} \cos x-0,457 e^{y} \sin x-$ $-0,345 e^{-y} \cos x-0,767 e^{\sqrt{2} y} \cos \sqrt{2} x+0,294 e^{\sqrt{2} y} \sin \sqrt{2} x$;

$\sigma_{y}^{(1)}(x, y)=-0,187 e^{y} \cos x+0,062 e^{y} \sin x-0,020 e^{-y} \cos x+0,045 e^{-y} \sin x-0,532 e^{\sqrt{2} y} \cos \sqrt{2} x-$ $-1,63 e^{\sqrt{2} y} \sin \sqrt{2} x-0,391 e^{-\sqrt{2} y} \cos \sqrt{2} x-0,181 e^{-\sqrt{2} y} \sin \sqrt{2} x+0,452 e^{\pi y} \cos \pi x-$ $-0,025 e^{\pi y} \sin \pi x+0,072 e^{-\pi y} \cos \pi x-0,087 e^{-\pi y} \sin \pi x+0,644 y e^{y} \cos x-$ $-0,028 y e^{y} \sin x-0,639 y e^{-y} \cos x-0,300 y e^{-y} \sin x+0,335 y e^{\sqrt{2} y} \cos \sqrt{2} x+$ $+1,22 y e^{\sqrt{2} y} \sin \sqrt{2} x+3,71 y e^{-\sqrt{2} y} \cos \sqrt{2} x-1,13 y e^{-\sqrt{2} y} \sin \sqrt{2} x-0,409 y e^{\pi y} \cos \pi x+$ $+0,021 y e^{\pi y} \sin \pi x-0,252 y e^{-\pi y} \cos \pi x+2,26 y e^{-\pi y} \sin \pi x$;

$u^{(2)}(x, y)=0,849 \cdot 10^{-12} e^{y} \sin x-0,229 \cdot 10^{-11} e^{y} \cos x-0,135 \cdot 10^{-10} e^{\sqrt{2} y} \sin \sqrt{2} x-$ $-0,510 \cdot 10^{-11} e^{\sqrt{2} y} \cos \sqrt{2} x-0,174 \cdot 10^{-12} e^{\pi y} \sin \pi x+0,171 \cdot 10^{-11} e^{\pi y} \cos \pi x-$ $-0,250 \cdot 10^{-12} y e^{y} \sin x-0,232 \cdot 10^{-11} y e^{y} \cos x-0,202 \cdot 10^{-10} y e^{\sqrt{2} y} \sin \sqrt{2} x+$ $+0,238 \cdot 10^{-11} y e^{\sqrt{2} y} \cos \sqrt{2} x+0,198 \cdot 10^{-11} y e^{\pi y} \sin \pi x+0,500 \cdot 10^{-11} y e^{\pi y} \cos \pi x$; 


$$
\begin{aligned}
v^{(2)}(x, y)= & -0,120 \cdot 10^{-11} e^{y} \cos x+0,106 \cdot 10^{-11} e^{y} \sin x-0,649 \cdot 10^{-11} e^{\sqrt{2} y} \cos \sqrt{2} x- \\
& -0,746 \cdot 10^{-11} e^{\sqrt{2} y} \sin \sqrt{2} x+0,105 \cdot 10^{-11} e^{\pi y} \cos \pi x-0,518 \cdot 10^{-12} e^{\pi y} \sin \pi x+ \\
& +0,250 \cdot 10^{-12} y e^{y} \cos x-0,232 \cdot 10^{-11} y e^{y} \sin x+0,202 \cdot 10^{-10} y e^{\sqrt{2} y} \cos \sqrt{2} x+ \\
& +0,238 \cdot 10^{-11} y e^{\sqrt{2} y} \sin \sqrt{2} x-0,198 \cdot 10^{-11} y e^{\pi y} \cos \pi x+0,500 \cdot 10^{-11} y e^{\pi y} \sin \pi x ; \\
\sigma_{x}^{(2)}(x, y)= & 0,117 e^{y} \cos x+0,729 e^{y} \sin x-6,36 e^{\sqrt{2} y} \cos \sqrt{2} x+0,955 e^{\sqrt{2} y} \sin \sqrt{2} x+ \\
& +0,187 e^{\pi y} \cos \pi x-1,69 e^{\pi y} \sin \pi x-0,045 y e^{y} \cos x+0,418 y e^{y} \sin x- \\
& -5,15 y e^{\sqrt{2} y} \cos \sqrt{2} x-0,607 y e^{\sqrt{2} y} \sin \sqrt{2} x+1,12 y e^{\pi y} \cos \pi x-2,83 y e^{\pi y} \sin \pi x ; \\
\tau_{x y}^{(2)}(x, y)= & 0,162 e^{y} \sin x-0,310 e^{y} \cos x-2,72 e^{\sqrt{2} y} \sin \sqrt{2} x-1,38 e^{\sqrt{2} y} \cos \sqrt{2} x- \\
& -0,169 e^{\pi y} \sin \pi x+0,788 e^{\pi y} \cos \pi x-0,045 y e^{y} \sin x-0,418 y e^{y} \cos x- \\
& -5,15 y e^{\sqrt{2} y} \sin \sqrt{2} x+0,607 y e^{\sqrt{2} y} \cos \sqrt{2} x+1,12 y e^{\pi y} \sin \pi x+2,83 y e^{\pi y} \cos \pi x ; \\
\sigma_{y}^{(2)}(x, y)= & -0,207 e^{y} \cos x+0,108 e^{y} \sin x-0,923 e^{\sqrt{2} y} \cos \sqrt{2} x-1,81 e^{\sqrt{2} y} \sin \sqrt{2} x+ \\
& +0,525 e^{\pi y} \cos \pi x-0,113 e^{\pi y} \sin \pi x+0,045 y e^{y} \cos x-0,418 y e^{y} \sin x+ \\
& +5,15 y e^{\sqrt{2} y} \cos \sqrt{2} x+0,607 y e^{\sqrt{2} y} \sin \sqrt{2} x-1,12 y e^{\pi y} \cos \pi x+2,83 y e^{\pi y} \sin \pi x .
\end{aligned}
$$

Графики функций при различных значениях $y$ приведены на рис. 1-6.


Р и с. 2 


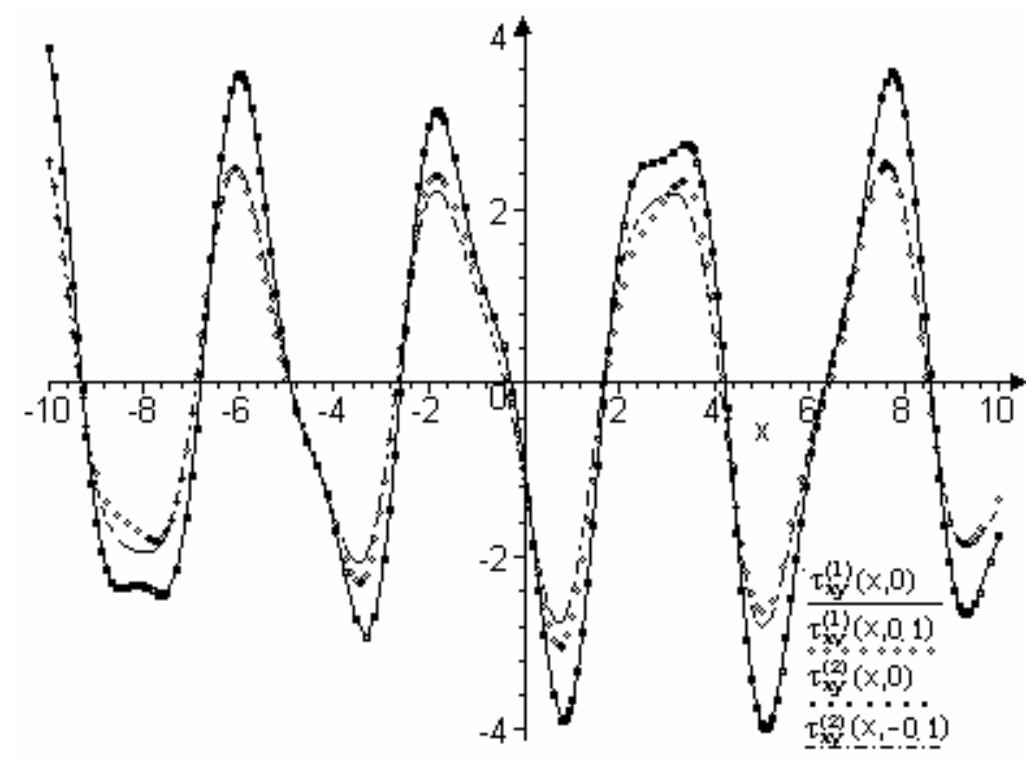

Р и с. 3

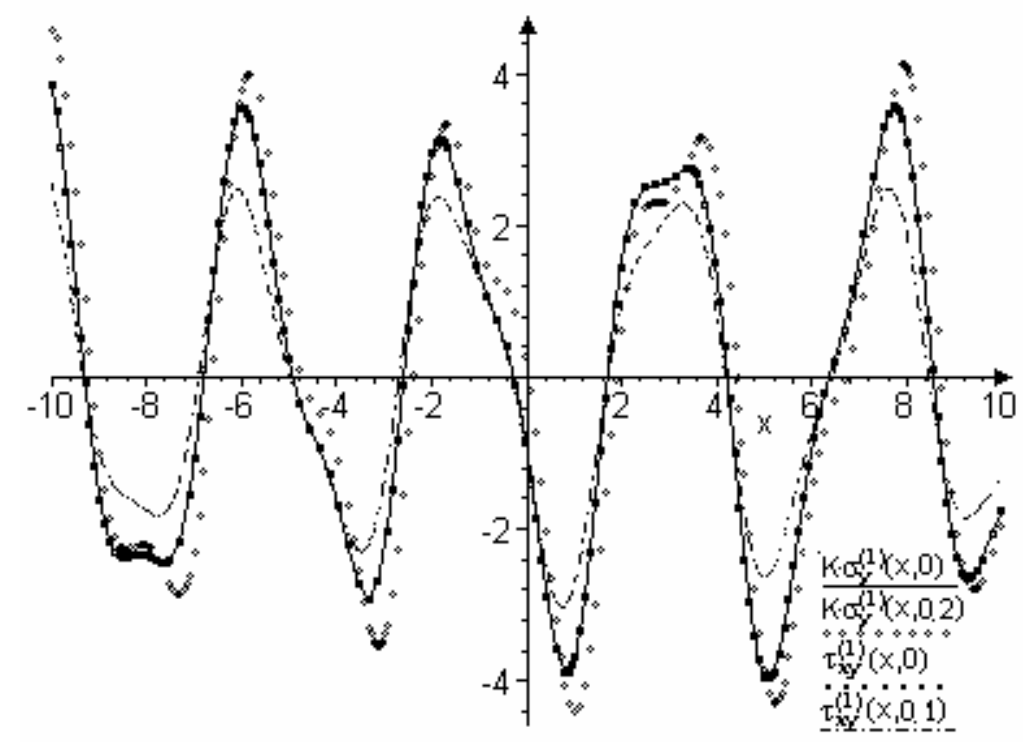

Р и с. 4

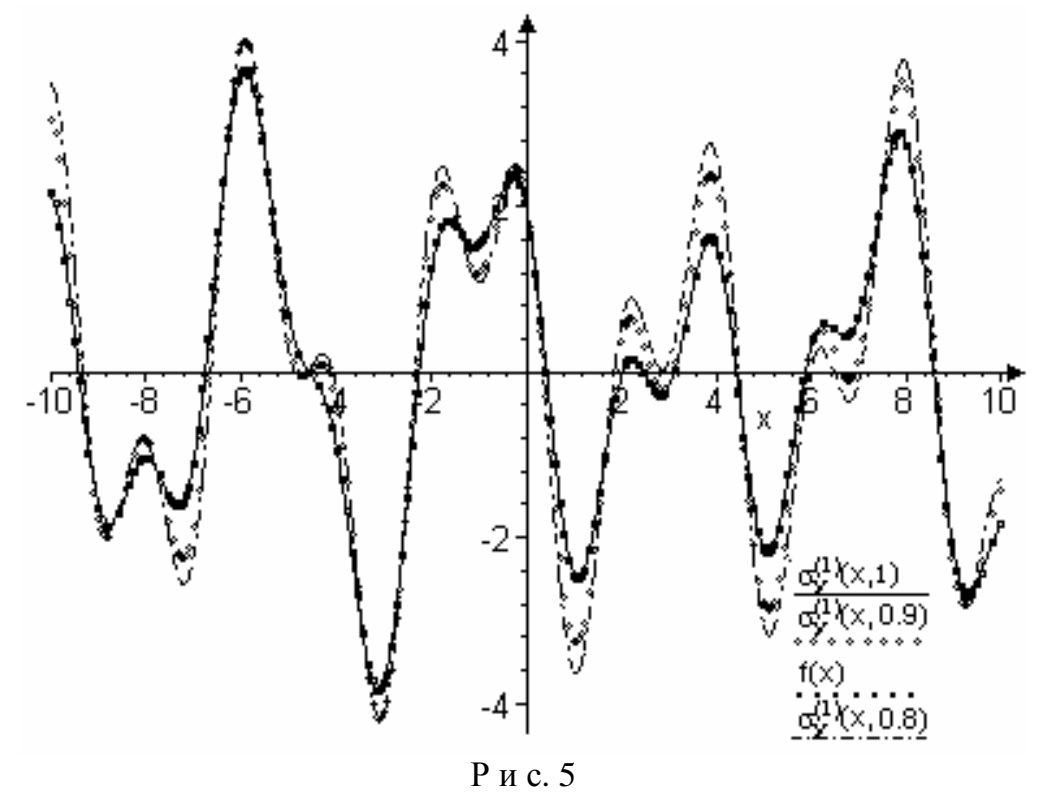




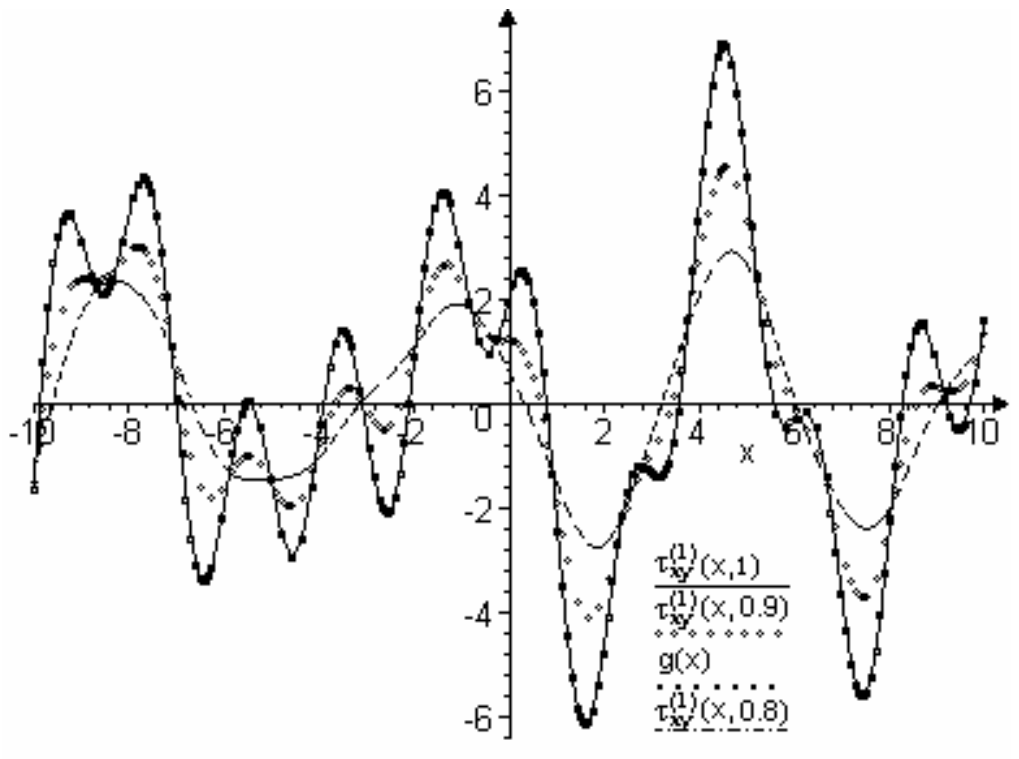

Р и с. 6

\section{БИБЛИОГРАФИЧЕСКИЙ СПИСОК}

1. К Кулагина М.Ф. О некоторых бесконечных системах с разностными индексами // Изв. вузов. Математика. 1992. №3. C. 18-23.

2. Кулагина М.Ф. Об интегральных уравнениях в средних значениях в пространствах почти-периодических функций // Изв. вузов. Математика. 1993. №8. С. 19-29.

3. Гохберг И.П., Крупник Н.Л. Введение в теорию одномерных сингулярных интегральных операторов. Кишинев: Штиинца, 1973. $423 \mathrm{c}$.

4. Левитан Б.М. Почти-периодические функции. М.: ГИТТЛ, 1953. 396 с.

5. $\quad$ Уфлянд Л.С. Интегральные преобразования в задачах теории упругости. Л.: Наука, 1968. 406 с.

Работа выполнена при финансовой поддержке Российского фонда фундаментальных исследований (проект 01-01-00720).

Поступила 6.03.2003 г. 